Bundesgesundheitsbl -

Gesundheitsforsch - Gesundheitsschutz

2000 • 43:400-406 @ Springer-Verlag 2000

Leitthema: Bundes-Gesundheitssurvey

E. Hermann-Kunz

Robert Koch-Institut, Berlin

\title{
Allergische Krankheiten in Deutschland
}

\section{Ergebnisse einer repräsentativen Studie}

\begin{abstract}
Schlüsselwörter
Lebenszeitprävalenz · Jahresprävalenz . Erwachsene, 0st- und Westdeutschland . Asthma - Allergische Rhinitis · Atopische Dermatitis · Allergisches Kontaktekzem . Urtikaria · Nahrungsmittelallergie . Kombinationshäufigkeiten allergischer Krankheiten
\end{abstract}

mit einem Selbstausfüllfragebogen und einem persönlichen, ärztlichen Interview erhoben. Die Übereinstimmung zwischen Fragebogen und Interviewdaten beträgt über $90 \%$ bei allergischer Rhinitis, Nahrungsmittelallergie und Urtikaria bzw. rund $80 \%$ bei allergischem Kontaktekzem und Asthma. Die Auswertungen beider Erhebungsmethoden ergaben deutliche 0st West-Unterschiede. Nach den Interviewdaten haben/hatten z.B. $17 \%$ aller westdeutschen, aber nur $11 \%$ aller ostdeutschen Erwachsenen eine allergische Rhinitis. Vergleichbare Unterschiede existieren bei allergischem Kontaktekzem. Viele Allergiker leiden bzw. litten an mehreren allergischen Krankheiten. Häufige Kombinationsmuster stellen Nahrungsmittelallergie/Heuschnupfen und Asthma/Heuschnupfen dar. Ein Vergleich mit früheren Studien zeigt, dass die Häufigkeit der allergischen Rhinitis von 1990/92 bis 1998 insgesamt um 70 \% gestiegen ist. Die relative Zunahme ist im Osten höher als im Westen. Bei Asthma konnte die zeitliche Entwicklung der Prävalenz nur für die westdeutsche Bevölkerung ermittelt werden. Die Ergebnisse zeigen auch hier einen deutlichen Anstieg.

\begin{abstract}
Yrankheiten des allergischen Formenkreises rücken in den letzten Jahrzehnten immer mehr in das öffentliche Interesse, da eine weite Verbreitung und kontinuierliche Zunahme dieser Krankheiten vermutet wird. Erste Ergebnisse des Bundes-Gesundheitssurveys 1998 unterstützen diese Annahme. In den letzten sechs bis acht Jahren ist beispielsweise die Häufigkeit der allergischen Rhinitis von rund $10 \%$ auf $17 \%$ gestiegen [1]. Fasst man alle im Bundes-Gesundheitssurvey erfragten, ärztlich diagnostizierten Krankheiten des allergischen Formenkreises zusammen, ergibt sich eine Lebenszeitprävalenz von $40 \%$. Das heißt, jeder zweite bis dritte Erwachsene hat im Laufe seines bisherigen Lebens schon ein- oder mehrmals über kürzere oder längere Zeit an einer Allergie gelitten [2]. Bei den bisher auf der Basis des Bundes-Gesundheitssurveys ausgewerteten Morbiditätsraten handelt es sich um Angaben zu ärztlich festgestellten Allergien. Es kann jedoch davon ausge-
\end{abstract}

gangen werden, dass zumindest bei einigen Allergieformen ein Teil der Betroffenen keinen Arzt aufsucht und eine Diagnose folglich nicht gestellt wird. Die tatsächliche Prävalenz wäre in diesen Fällen also höher, als die bisher veröffentlichten Angaben.

\section{„Jeder zweite bis dritte Erwachsene hat im Laufe seines bisherigen Lebens schon ein-oder mehrmals aneiner Allergie gelitten."}

In diesem Beitrag werden deshalb die Ergebnisse des ärztlichen Interviews mit den Fragebogendaten zur Lebenszeitprävalenz allergischer Krankheiten verglichen. Zur Beurteilung des Krankheitsgeschehens und der Krankheitslast in einer Bevölkerung sind neben den Angaben zur Lebenszeitprävalenz aber auch die aktuellen Krankheitszahlen von Bedeutung. Um den Bevölkerungsanteil akut Betroffener zu ermitteln, wurde die Morbiditätsrate der letzten zwölf Monate berechnet. In der Epidemiologie allergischer Erkrankungen wird dieser Zeitraum üblicherweise betrachtet, um der Saisonalität dieser Krankheiten Rechnung zu tragen. Da Allergiker im Laufe ihres Lebens häufig

Dr. E. Hermann-Kunz Robert Koch-Institut, Postfach 650280, 13302 Berlin 


\section{E. Hermann-Kunz}

\section{Allergic diseases in Germany}

\section{Summary}

The results of the German National Health Interview and Examination Survey 1998 confirm allergies as widespread diseases in Germany. In total 40 to $43 \%$ of the adult population have or have had an allergic disease. The morbidity was assessed using a self-administered questionnaire and a personal interview performed by a physician. The agreement between questionnaire and interview data is greater than $90 \%$ for allergic rhinitis, food allergy and urticaria and about $80 \%$ for contact dermatitis and asthma. The results of both assessment methods showed remarkable differences between East and West Germany. Using the interview data $17 \%$ of the West but only 11 $\%$ of the East German adults have/had e.g. an allergic rhinitis. Comparable differences exist for contact dermatitis. Many people with allergies suffer or have suffered from several allergies. Frequent patterns of combination are food allergy/allergic rhinitis and asthma/allergic rhinitis. In comparison with former studies we observed an increase of $70 \%$ in the prevalence of allergic rhinitis between 1990/92 and 1998. The relative increase is higher in East than in West. The time trend in prevalence of asthma could only be calculated for the West German population. The results show a remarkable increase here, too.

\section{Keywords}

Lifetime prevalence - Prevalence in the last 12 months · Adults, East and West Germany . Asthma - Allergic rhinitis - Atopic dermatitis, Contact dermatitis - Urticaria - Food allergy . Frequency of combination of allergic diseases nicht nur an einer spezifischen Allergie leiden, sondern weitere Manifestationsformen hinzutreten, bzw. Kreuzreaktionen z.B. bei Pollen- und Nahrungsmittelallergien existieren $[3,4]$, wurde zusätzlich untersucht, wie häufig bei den Betroffenen die verschiedenen Kombinationen allergischer Erkrankungen vorkommen.

\section{Material und Methoden}

Im Bundes-Gesundheitssurvey 1998 wurden zur Erfassung der Morbidität zwei Methoden eingesetzt: ein Interview, das von einem Arzt durchgeführt wurde, und ein von den Probanden selbst auszufüllender Fragebogen. Erhoben wurden mit beiden Methoden folgende Allergien:

D Asthma bronchiale (Bronchialasthma, Lungenasthma, allergisches Asthma),

D Heuschnupfen, allergische Bindehautentzündung (allergische Rhinitis, allergische Konjunktivitis),

D allergisches Kontaktekzem (Hautausschlag durch Waschmittel, Kosmetika, Nickel, Unverträglichkeit anderer Metalle u.a.),

D Neurodermitis (juckendes Ekzem, besonders in den Ellenbeugen und Kniekehlen, endogenes Ekzem, atopisches Ekzem),

D Nahrungsmittelallergie,

D allergische Hautquaddeln (flüchtige, stark juckende, schubweise aufschießende Quaddeln, Nesselsucht, Urtikaria),

D sonstige Allergien.

Im Selbstausfüll-Fragebogen gaben die Probanden an, welche der genannten Krankheiten sie jemals hatten. In dem ärztlichen Interview wurde erfragt, ob ein Arzt jemals eine der genannten Krankheiten oder Gesundheitsstörungen festgestellt hat. Das ärztliche Interview enthielt noch ergänzende Fragen u.a. zum Zeitpunkt des letztmaligen Auftretens der Erkrankung. Insgesamt nahmen 7099 Probanden an dem ärztlichen Interview teil, und 6974 Personen füllten den Fragebogen aus. Die Ausgangsstichprobe des Bundes-Gesundheitssurveys ist eine repräsentative Stichprobe der 18- bis 79-jährigen Wohnbevölkerung in Deutschland. Die Repräsentativität bezieht sich auf die Merkmale Bundesland, Gemeindegrö- ßenklasse, Geschlecht und Alter. Wie bei allen Untersuchungen mit freiwilligen Teilnehmern weicht die realisierte, d.h. die Nettostichprobe, zum Teil von der ursprünglich angestrebten Verteilung ab. Um dennoch weitgehend repräsentative Aussagen für die erwachsene Wohnbevölkerung treffen zu können, wurde bis auf wenige, extra gekennzeichnete Ausnahmen bei der Datenanalyse ein Gewichtungsfaktor verwendet. Eine ausführliche Beschreibung des Surveys sowie nähere Informationen zur Stichprobenauswahl, zur Zusammensetzung der Stichprobe, zum Gewichtungsfaktor und zu den Responseraten sind den Publikationen Bellach et al.1998 [5] und Thefeld et al. 1999 [6] zu entnehmen. Zur Untersuchung der zeitlichen Prävalenzentwicklung wurden Daten der Nationalen Untersuchungssurveys der Deutschen Herz-Kreislauf-Präventionsstudie (DHP) von 1984/86 bis 1990/91 und Daten des Gesundheitssurveys Ost von 1991/92 herangezogen [7]. Auch diese Auswertungen wurden gewichtet durchgeführt. Die Gewichtungsfaktoren unterscheiden sich jedoch u.a. von den erstgenannten dadurch, dass in den früheren Untersuchungen keine Ausländer mit erfasst wurden und die Alterszusammensetzung der DHP auf Personen von 25 bis 69 Jahren beschränkt war. Die Datenanalysen wurden mit der Statistik-Software SAS Version 6.12 durchgeführt.

\section{Ergebnisse}

\section{Lebenszeitprävalenz ärztlich diagnostizierter Allergien}

Rund 15\% aller Erwachsenen haben oder hatten schon einmal einen allergischen Schnupfen oder ein allergisches Kontaktekzem. Bei etwa $6 \%$ aller 19- bis 79-Jährigen wurde schon einmal eine Nahrungsmittelallergie oder Asthma bronchiale festgestellt und bei ca. 3\% eine Neurodermitis. Die Prävalenz aller allergischen Krankheiten ist, bis auf die Neurodermitis, im Osten statistisch signifikant niedriger als im Westen (s. Tabelle 1). Ein allergisches Kontaktekzem haben beispielsweise $16 \%$ aller west- und $11 \%$ aller ostdeutschen Erwachsenen, die Prävalenz der allergischen Rhinitis beträgt rund $17 \%$ im Westen und ebenfalls nur $11 \%$ im Osten. 


\section{Leitthema: Bundes-Gesundheitssurvey}

Tabelle 1 Lebenszeitprävalenz allergischer Krankheiten (in Prozent). Ergebnisse der ärztlichen Befragung

\begin{tabular}{|c|c|c|c|c|c|c|c|c|c|c|c|c|c|}
\hline \multirow{3}{*}{ Allergische Rhinitis } & \multicolumn{3}{|c|}{ Deutschland } & \multicolumn{4}{|c|}{ Gesamt } & \multicolumn{3}{|c|}{ Männer } & \multicolumn{3}{|c|}{ Frauen } \\
\hline & Gesamt & West & Ost & & Männer & Fra & & West & Ost & & West & Ost & \\
\hline & 15 & 17 & 11 & $* * *$ & 14 & 16 & * & 16 & 10 & $* * *$ & 18 & 11 & *** \\
\hline Allerg. Kontaktekzem- & 15 & 16 & 11 & $* * *$ & 8 & 21 & $* * *$ & 9 & 5 & $* *$ & 22 & 17 & ** \\
\hline Urtikaria & 8 & 8 & 5 & $* * *$ & 5 & 11 & $* * *$ & 5 & 3 & $* *$ & 11 & 8 & ** \\
\hline $\begin{array}{l}\text { Nahrungsmittel- } \\
\text { allergie }\end{array}$ & 6 & 6 & 3 & $* * *$ & 4 & 8 & $* * *$ & 4 & 2 & * & 8 & 4 & *** \\
\hline Asthma bronchiale & 6 & 6 & 4 & *** & 5 & 6 & * & 5 & 4 & & 7 & 4 & ** \\
\hline Neurodermitis & 3 & 4 & 3 & ns & 3 & 4 & $* * *$ & 3 & 3 & ns & 5 & 2 & ** \\
\hline Sonstige Allergien & 15 & 17 & 9 & $* * *$ & 12 & 18 & $* * *$ & 13 & 6 & $* * *$ & 20 & 11 & $* * *$ \\
\hline $\begin{array}{l}\text { mindestens eine } \\
\text { der gen. Allergien }\end{array}$ & 40 & 43 & 29 & $* * *$ & 33 & 47 & $* * *$ & 35 & 23 & $* * *$ & 50 & 36 & $* *$ \\
\hline $\begin{array}{l}\text { mindestens eine } \\
\text { der gen. Allergien } \\
\text { nach den Fragebogen- } \\
\text { daten }\end{array}$ & 43 & 45 & 33 & $* * *$ & 35 & 49 & $* * * *$ & 38 & 27 & $* * *$ & 52 & 38 & $* * *$ \\
\hline
\end{tabular}

$* * *=p=0,001 ; * *=p=0,01 ; *=p=0,05 ; n s=p>0,05\left(\right.$ Chi' ${ }^{2}$-Test $)$

\section{"Die Prävalenz aller allergischen Krankheiten ist - bis auf die Neurodermitis - im Osten statistisch signifikant niedriger als im Westen."}

Insgesamt sind Frauen signifikant häufiger von Allergien betroffen als Männer. Der Unterschied zwischen den Geschlechtern ist bei dem allergischen Kontaktekzem, der Urtikaria und der Nahrungsmittelallergie besonders hoch. Im Vergleich zu Männern haben Frauen bei den genannten Krankheiten eine zwei- bis fast dreifach höhere Prävalenz. Am geringsten ist die Differenz bei der allergischen Rhinitis und beim Asthma bronchiale. Nach Ost- und Westdeutschland stratifiziert, ergibt der Vergleich zwischen Frauen und Männern nur bei der Neurodermitis ein gegenläufiges Ergebnis, im Westen sind Frauen fast doppelt so häufig betroffen wie Männer, im Osten ist die Morbidität dagegen bei Männern höher. Zusammengefasst haben $33 \%$ aller Männer und $47 \%$ aller Frauen in Deutschland irgendwann in ihrem Leben schon einmal an einer ärztlich diagnostizierten Allergie gelitten

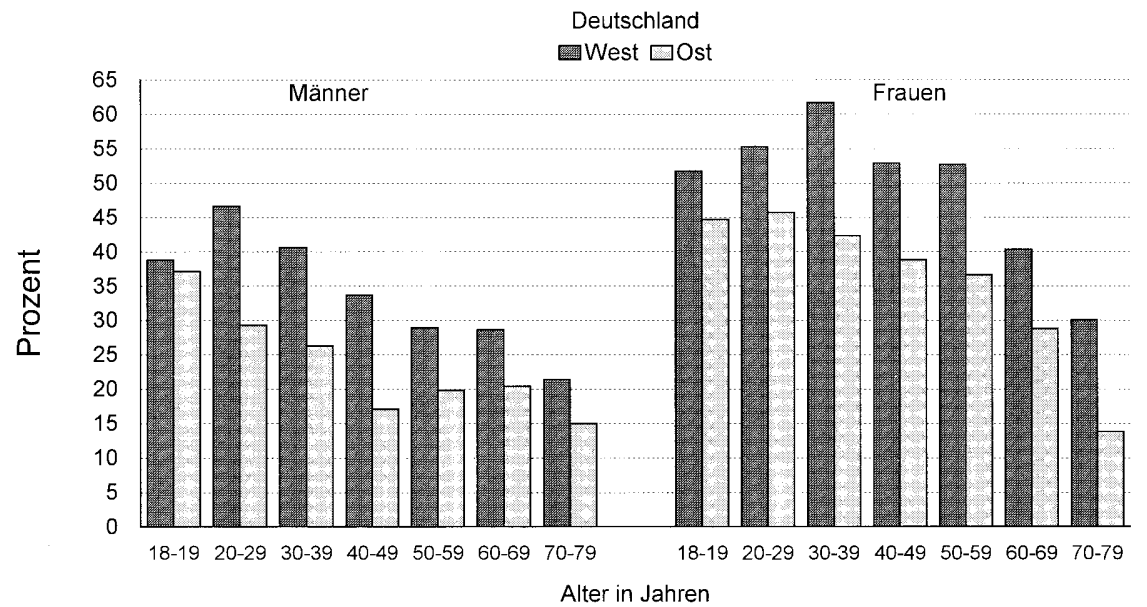

Abb. 1 Lebenszeitprävalenz mindestens einer allergischen Krankheit nach Altersgruppen. Ergebnisse der ärztlichen Befragung

(Tabelle 1). Mit zunehmendem Alter sinkt die Allergiehäufigkeit, die Prävalenzraten sind jedoch auch bei den 60bis 80 -Jährigen noch hoch (s.Abb.1). Die Morbiditätsrate beträgt beispielsweise bei 60 - bis 69 -jährigen Männern 20 bis $30 \%$ und bei Frauen dieser Altersgruppe 30 bis $40 \%$. Aus Abb. 1 geht hervor, dass westdeutsche Frauen im Alter von 30 bis 39 Jahren besonders häufig mindestens eine allergische Krankheit haben oder hatten (62\%). Einen großen Anteil an dieser Prävalenz haben allergische Kontaktekzeme. Sie treten mit einer Häufigkeit von 33\% bei den genannten Frauen auf.

\section{Lebenszeitprävalenz nach den Fragebogendaten}

Ein Vergleich der Fragebogendaten mit den Ergebnissen des ärztlichen Interviews ist in Abb. 2 dargestellt. Mit Ausnahme der sonstigen Allergien ${ }^{*}$ ist bei allen anderen allergischen Erkrankungen die Lebenszeitprävalenz nach den Fragebogendaten höher als die ärztlich diagnostizierten Allergien. Die Unterschiede sind bei den meisten Manifestationsformen allerdings gering. Zu über

\footnotetext{
*Unter dem Sammelbegriff sonstiger Allergie wurden u.a. Allergien auf Insektenstiche und Arzneimittel zusammengefasst.
} 


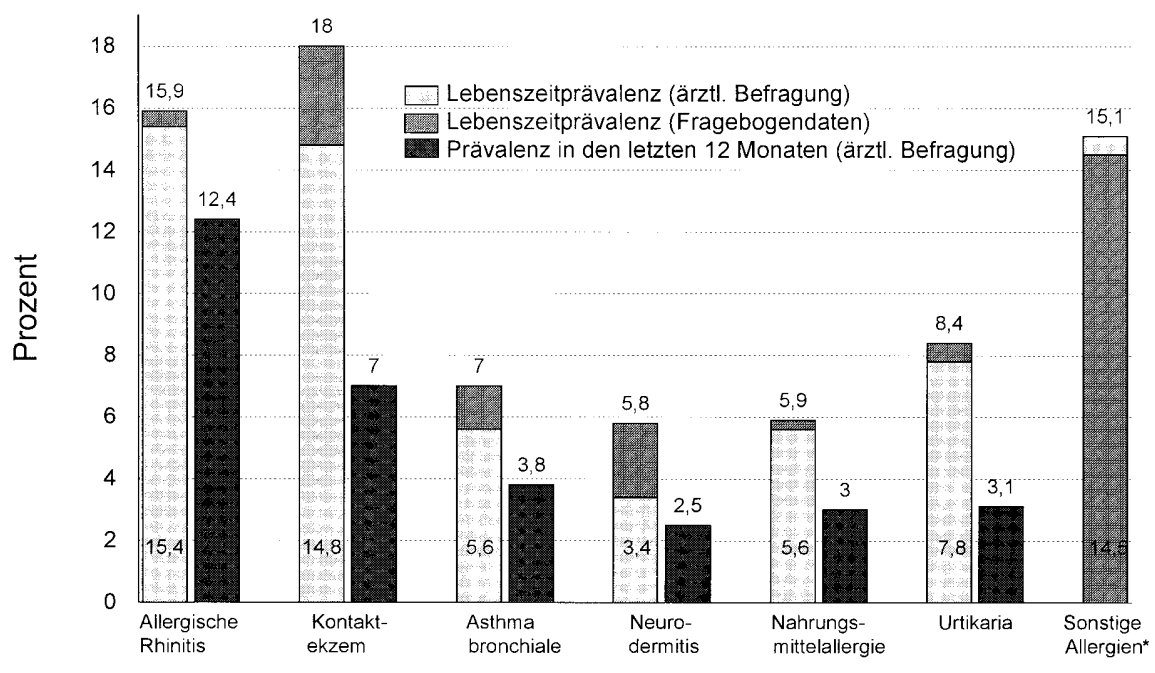

* Die Prävalenz in den letzten 12 Monaten konnte nicht dargestellt werden, da bei den sonstigen Allergien der Zeitpunkt des letzten Auftretens nic erfragt wurde.

Abb.2 $\triangle$ Prävalenz allergischer Krankheiten. Lebenszeitprävalenz und Prävalenz in den letzten zwölf Monaten

90\% stimmen die Ergebnisse der beiden Befragungsmethoden z.B. bei der allergischen Rhinitis, der Nahrungsmittelallergie und der Urtikaria überein. Bei einem allergischen Kontaktekzem und bei Asthma bronchiale gaben etwa $80 \%$ aller Betroffenen eine ärztlich bestätigte Diagnose an. Deutlich geringer ist die Übereinstimmung dagegen bei der Neurodermitis, hier wurden nur ca. $60 \%$ aller Erkrankungen ärztlich festgestellt.

$\mathrm{Da}$ die Allergieformen mit den höchsten Prävalenzraten auch die größte Übereinstimmung bei beiden Erhebungsmethoden aufweisen, ergeben sich bei der zusammengefassten Berechnung der Lebenszeitprävalenz aller betrachteten Manifestationsformen wenig Unterschiede $\mathrm{zu}$ den bereits dargestellten Häufigkeiten der ärztlich bestätigten Erkrankungen. Die auf den Fragebogendaten basierende kumulative Prävalenz mindestens einer Allergie beträgt insgesamt $43 \%$, die entsprechende Rate nach den ärztlichen Interviews liegt bei $40 \%$ (Tabelle 1). Die Differenz zwischen Ostund Westdeutschland sowie zwischen Frauen und Männern spiegelt sich in den Fragebogen- und in den Interviewdaten ebenfalls in vergleichbarer Höhe wider.

\section{Prävalenz in den letzten zwölf Monaten}

Die aktuelle Prävalenz allergischer Krankheiten, d.h. die Prävalenz in den letzten zwölf Monaten ist ebenfalls in einer genetischen Prädisposition beruärztlichen Befragung einer ärztlich festgestellten atopischen Erkrankung trat die Allergie auch in den letzten zwölf Monaten auf. An Nahrungsmittelallergien, Urtikaria und allergischem Kontaktekzem haben dagegen nur noch 40 bis 50\% der Betroffenen im Laufe des letzten Jahres gelitten. Ein Grund hierfür dürfte darin liegen, dass z.B. bei einer bekannten Überempfindlichkeit auf bestimmte Lebensmittel oder bei bekannter Ursache eines Kontaktekzems eine Expositionsvermeidung durchgeführt werden kann. Bei den atopischen Erkrankungen ist eine Allergenkarenz in der Regel nicht so einfach, weil entweder das krankmachende Agens nicht bekannt ist oder die Exposition auch bei bekanntem Agens nicht oder kaum vermieden werden kann (z.B. Pollenallergie).

\section{Gemeinsames Auftreten allergischer Krankheiten}

Abb. 2 dargestellt. Ein Vergleich der aktuellen und der Lebenszeitprävalenz kann nur auf der Grundlage der ärztlichen Interviews erfolgen, da im Fragebogen der Zeitpunkt des letzten Auftretens nicht erhoben wurde. Bei den klassischen atopischen Krankheiten, wie Neurodermitis, Rhinitis allergica und Asthma bronchiale, bei denen eine auf hende klinische Manifestation einer Überempfindlichkeitsreaktion vom Soforttyp (Typ I) vorliegt, sind die Unterschiede zwischen ärztlich bestätigter Lebenszeit- und aktueller Prävalenz geringer als bei den anderen Allergieformen. Bei rund 70 bis $80 \%$ aller Probanden mit
Allergiker leiden häufig nicht nur an einer allergischen Krankheit, sondern im Laufe ihres Lebens kommen weitere Allergieformen hinzu. Aus der Literatur ist bekannt, dass z.B. Patienten mit einer Neurodermitis verstärkt dazu neigen, auch ein allergisches Kontaktekzem zu entwickeln und Patienten mit Kontaktekzem berichten, dass sie im Laufe der Zeit auf immer mehr Substanzen allergisch reagieren. Bekannt ist auch der Zusammenhang zwischen den atopischen Krankheiten Asthma bronchiale, Heuschnupfen und Neurodermitis. Bei einem relativ hohen Prozentsatz von Heu-

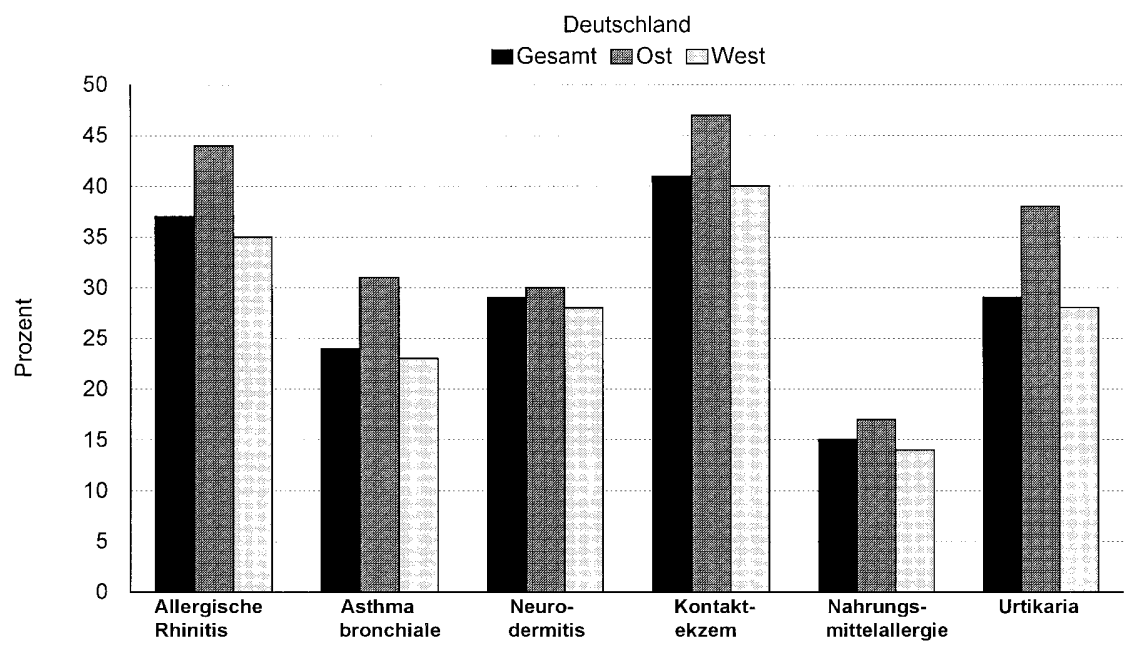

Abb. 3 Prozentualer Anteil allein auftretender allergischer Erkrankungen. Ergebnisse der 


\section{Leitthema: Bundes-Gesundheitssurvey}

Tabelle 2

Kombinationshäufigkeiten allergischer Erkrankungen (in Prozent)* . Ergebnisse der ärztlichen Befragung

\begin{tabular}{|c|c|c|c|c|c|c|}
\hline $\begin{array}{l}\text { mit allerg. } \\
\text { Rhinitis }\end{array}$ & $\begin{array}{l}\text { mit } \\
\text { Asthma } \\
\text { bronchiale }\end{array}$ & $\begin{array}{l}\text { mit allerg. } \\
\text { Kontakt- } \\
\text { ekzem }\end{array}$ & $\begin{array}{l}\text { mit Neuro- } \\
\text { dermitis }\end{array}$ & $\begin{array}{l}\text { mit Nah- } \\
\text { rungsmittel- } \\
\text { allergie }\end{array}$ & $\begin{array}{l}\text { mit } \\
\text { Urtikaria }\end{array}$ & $\begin{array}{l}\text { mit } \\
\text { sonst. } \\
\text { Allergie }\end{array}$ \\
\hline
\end{tabular}

\section{Allergische Rhinitis}

$\begin{array}{lllllll}\text { Gesamt } & 19 & 27 & 8 & 21 & 17 & 27 \\ \text { West } & 21 & 29 & 9 & 23 & 18 & 28 \\ \text { Ost } & 15 & 24 & 8 & 14 & 12 & 23\end{array}$

Asthma bronchiale

$\begin{array}{lllllll}\text { Gesamt } & 52 & 29 & 12 & 21 & 19 & 32 \\ \text { West } & 55 & 30 & 14 & 24 & 20 & 35 \\ \text { Ost } & 41 & 27 & 6 & 11 & 14 & 22\end{array}$

Allerg. Kontaktekzem

$\begin{array}{lll}\text { Gesamt } & 28 & 11 \\ \text { West } & 30 & 12 \\ \text { Ost } & 22 & 9\end{array}$

Ost

11

12

Neurodermitis

$\begin{array}{llll}\text { Gesamt } & 37 & 19 \\ \text { West } & 39 & 23 \\ \text { Ost } & 31 & 8\end{array}$

Nahrungsmittelallergie

\begin{tabular}{|c|c|c|c|c|c|c|c|}
\hline Gesamt & 58 & 22 & 43 & 15 & & 29 & 37 \\
\hline West & 60 & 24 & 41 & 14 & & 31 & 39 \\
\hline Ost & 47 & 13 & 50 & 16 & & 24 & 28 \\
\hline \multicolumn{8}{|l|}{ Urtikaria } \\
\hline Gesamt & 34 & 14 & 34 & 10 & 21 & & 31 \\
\hline West & 37 & 15 & 35 & 10 & 23 & & 33 \\
\hline Ost & 23 & 11 & 32 & 8 & 15 & & 26 \\
\hline \multicolumn{8}{|c|}{ Sonstige Allergie } \\
\hline Gesamt & 28 & 12 & 28 & 6 & 14 & 16 & \\
\hline West & 29 & 13 & 29 & 5 & 15 & 17 & \\
\hline 0st & 26 & 9 & 26 & 7 & 9 & 14 & \\
\hline
\end{tabular}

* ungewichtet berechnet der Anteil isoliert auftretender Allergien bei allen Manifestationsformen im Osten höher.

Welche Allergien wie häufig gemeinsam auftreten bzw. an welchen weiteren Allergien ein Proband irgendwann in seinem Leben zusätzlich erkrankte, ist in der Tabelle 2 dargestellt.

\section{„Allergiker leiden häufig nicht nur an einer allergischen Krankheit, im Laufe ihres Lebens kommen weitere Allergieformen hinzu."}

Etwa 30\% aller Personen mit einer allergischen Rhinitis haben oder hatten auch ein Kontaktekzem. Eine Nahrungsmittelallergie oder Asthma bronchiale besteht bzw. bestand bei ca. $20 \%$, ein atopisches Ekzem dagegen nur bei 8\% aller Erwachsenen mit Rhinitis allergica. Betrachtet man umgekehrt alle an Asthma bronchiale oder einer Nahrungsmittelallergie Erkrankten, stellt man fest, dass 52 bis $58 \%$ auch einen allergischen Schnupfen im ärztlichen Interview genannt haben. Eine häufige Kombination stellen weiterhin Asthma/Kontaktekzem (29\% der Asthmatiker) sowie Nahrungsmittelallergien/Kontaktekzem (43\% der Probanden mit Nahrungsmittelallergie) dar. Mit einer Prävalenz von 30 bis $40 \%$ ist bei Personen mit Neurodermitis und bei Personen mit Urtikaria ein allergischer Schnupfen und/oder ein allergisches Kontaktekzem diagnostiziert worden. Ebenfalls oft kombiniert treten die folgenden Manifestationen auf: Nahrungsmittelallergie/Urtikaria, Neurodermitis/Nahrungsmittelallergie und Neurodermitis/Urtikaria. Die Prävalenz dieser Kombinationen liegt bei ca. 20 bis $30 \%$. Bei den drei letztgenannten Kombinationsmustern handelt es sich aber möglicherweise nicht um unterschiedliche Allergien, sondern die Überempfindlichkeit gegen Nahrungsmittel könnte der Auslöser der Neurodermitis oder der Urtikaria sein.

\section{Zeitlicher Trend bei der Prävalenz von allergischer Rhinitis und Asthma bronchiale}

Die Schätzung der Prävalenzentwicklung bei der allergischen Rhinitis erfolgte auf der Grundlage der Fragebogenda- 


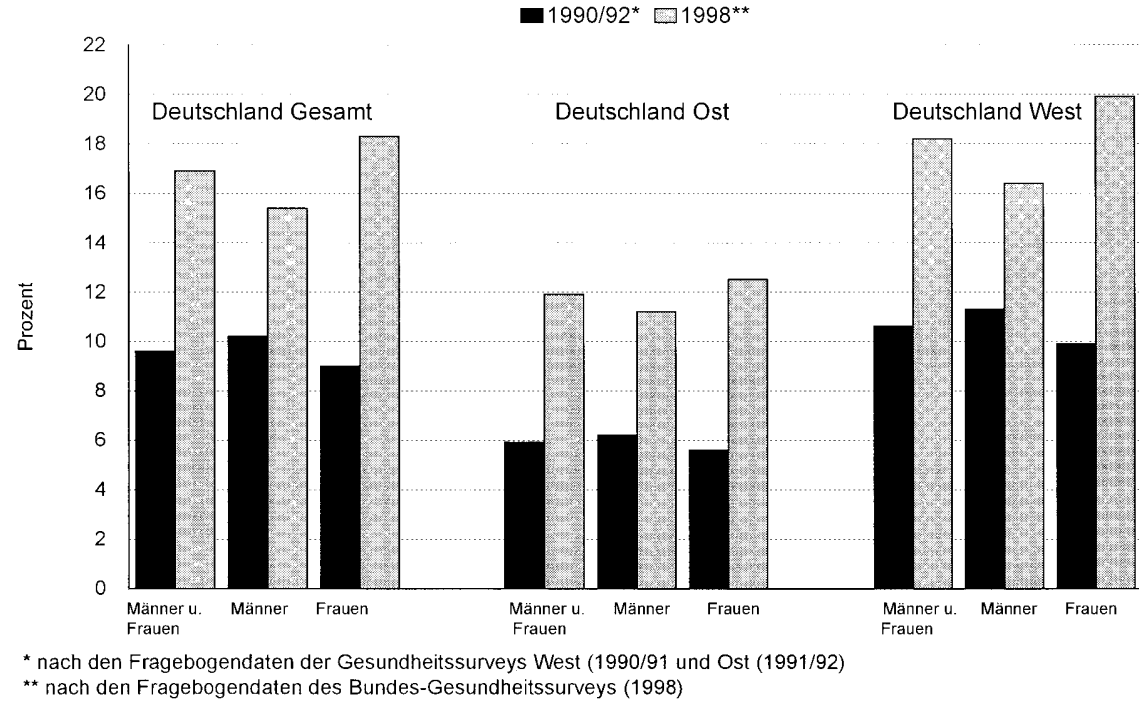

Abb.4 \ Allergische Rhinitis.Zeitliche Entwicklung der Prävalenz in 0st- und Westdeutschland

ten der Gesundheitssurveys in Ostdeutschland (1991/92) und in Westdeutschland (1990/91) sowie der Fragebogendaten des Bundes-Gesundheitssurveys 1998. Die Ermittlung der Trendentwicklung bei Asthma bronchiale basiert für die westdeutsche Bevölkerung auf den Fragebogendaten des BundesGesundheitssurveys und der Nationalen Untersuchungssurveys der DHP. Für die ostdeutsche Bevölkerung wurden Trendschätzungen aufgrund der unterschiedlichen Fragestellungen im Gesundheitssurvey Ost 1991/92 und im Bundes-Gesundheitssurvey nicht durchgeführt.

Bei Heuschnupfen ist von 1990/92 bis 1998 eine extrem hohe Morbiditäts- zunahme bei den 25- bis 69-jährigen Bundesbürgern zu verzeichnen. Wie aus der Abb. 4 zu ersehen ist, stiegen nach den Eigenangaben der Studienteilnehmer die Erkrankungsraten insgesamt um $70 \%$ (von ca. $10 \%$ auf $17 \%$ ).

Der relative Anstieg ist bei Frauen doppelt so hoch wie bei Männern (Frauen: von $9 \%$ auf $18 \%$; Männer: von ca. $10 \%$ auf $15 \%)$. Differenziert nach Ostund Westdeutschland ergibt sich, dass zwar die Prävalenz im Osten signifikant niedriger ist, die relative Prävalenzzunahme jedoch höher (Männer: West ca. $45 \%$, Ost ca. $80 \%$; Frauen: West ca. $100 \%$, Ost ca. $120 \%)$.

Basierend auf den Fragebogendaten hat sich die Häufigkeit von Asthma

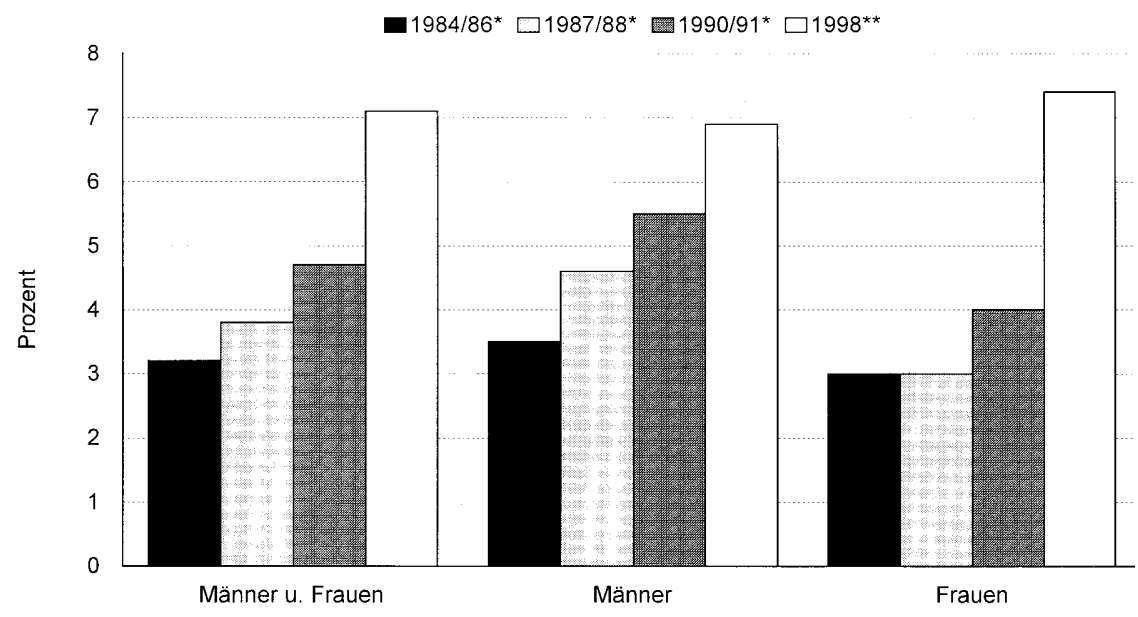

* Fragebogendaten der Nationalen Untersuchungssurveys der Deutschen Herz-Kreislauf-Präventionsstudie ** Fragebogendaten des Bundes-Gesundheitssurveys

Abb. 5 Asthma bronchiale. Zeitliche Entwicklung der Prävalenz in der westdeutschen Bevölkerung

bronchiale in den letzten 12 bis 14 Jahren mehr als verdoppelt, die Prävalenz stieg von $3 \%$ auf $7 \%$ (s. Abb. 5). Ein besonders steiler Anstieg ist von 1990/91 bis $1998 \mathrm{zu}$ verzeichnen. Auffällig ist hierbei die deutlich höhere Morbiditätszunahme bei Frauen ( $85 \%$ bei Frauen, $25 \%$ bei Männern). Die Höhe der Prävalenz (Männer 6,9\%; Frauen 7,4\%) unterscheidet sich dagegen bei Männern und Frauen nur geringfügig.

\section{Diskussion}

Studien, die kurz nach der Wiedervereinigung durchgeführt wurden, wiesen bereits auf eine geringere Prävalenz allergischer Erkrankungen in Ostdeutschland hin. Regionale Studien bei neunbis 11-jährigen Kindern ergaben z.B.kumulative Prävalenzen bei Heuschnupfen von $9 \%$ in München und $3 \%$ in Leipzig [8]. Repräsentative Aussagen zur Allergiehäufigkeit, die den Ost-West-Unterschied auch in der erwachsenen Bevölkerung bestätigen, waren erstmals auf der Datenbasis des Nationalen Untersuchungssurveys der DHP (1990/91) und des Gesundheitssurveys Ost (1991/92) möglich $[9,10,11]$. Die aktuellen Daten des Bundes-Gesundheitssurveys zeigen, dass Ostdeutsche nach wie vor seltener an einer Allergie leiden als Westdeutsche. Mit Ausnahme der Neurodermitis ist die Prävalenz bei allen allergischen Manifestationsformen im Osten statistisch signifikant niedriger als im Westen, und Frauen aus beiden Teilen des Landes erkranken deutlich häufiger an einer Allergie als Männer. Die beschriebenen Prävalenzen der einzelnen allergischen Krankheiten stimmen mit den Ergebnissen anderer aktueller Studien bei Erwachsenen gut überein. Vergleichsdaten liegen vor allem für Heuschnupfen und Asthma bronchiale vor. So ergab z.B. die Schweizer SAPALDIA-Studie, an der über 8000 Personen im Alter von 18 bis 60 Jahren teilnahmen, bei allergischer Rhinitis eine kumulative Prävalenz von rund $14 \%$ (15\% nach den Interviewdaten im Bundes-Gesundheitssurvey) und bei atopischem Asthma von $4 \%$ (6\% bei Asthma insgesamt im BundesGesundheitssurvey) [12]. In den European Community Respiratory Health Surveys wurden für allergische Rhinitis bei 20- bis 44-Jährigen u.a. folgende Prävalenzen ermittelt: Paris 31\%, Grenoble $28 \%$ [13], Uppsala $22 \%$ [14] und Ve- 
rona $17 \%$ [15]. Die Prävalenz im BundesGesundheitssurvey beträgt für diese Altersgruppe $20 \%$.

Bei dem Vergleich der Fragebogendaten mit den Ergebnissen des ärztlichen Interviews erweckt der Begriff "ärztlich diagnostizierte Allergie" den Eindruck, dass diese Daten valider sind und die Prävalenz allergischer Erkrankungen besser widerspiegeln. Unter dem Gesichtspunkt, dass Laien vorhandene Symptome fälschlicherweise auf eine Allergie zurückführen, die medizinisch gesehen nicht in den Formenkreis allergischer Krankheiten einzuordnen sind, stimmt diese Annahme. Da die Symptome allergischer Krankheiten von kaum wahrnehmbaren Erscheinungen ohne nennenswerte Beeinträchtigung der Lebensqualität bis zu schweren, chronischen - sogar lebensbedrohlichen - Manifestationsformen reichen, ist es jedoch durchaus denkbar, dass Personen mit leichteren Beschwerden keinen Arzt aufsuchen und eine ärztliche Diagnosestellung trotz vorhandener Allergie unterbleibt. Vorstellbar ist auch, dass das Allergie auslösende Agens von den Betroffenen selbst identifiziert wird und die Symptome durch die Vermeidung eines erneuten Kontaktes verhindert werden können. Auch in diesen Fällen ist nicht unbedingt damit $\mathrm{zu}$ rechnen, dass ein Arzt aufgesucht und die Diagnose bestätigt wird. Die Höhe der tatsächlichen Morbidität wird möglicherweise also eher durch die Fragebogendaten als durch das ärztliche Interview erfasst. Da die Unterschiede, wie dargestellt, in den meisten Fällen relativ gering sind, würden sich die Aussagen zur Häufigkeit allergischer Erkrankungen hierdurch jedoch nicht wesentlich ändern, eine Diskussion der verwendeten Datenbasis erscheint in diesem Zusammenhang bei der Mehrzahl allergischer Krankheiten deshalb nicht relevant. Anders ist die Differenz zwischen den beiden Erhebungsmethoden jedoch beim Asthma bronchiale zu sehen. Angenommen, dass Personen, die tatsächlich an Asthma leiden, aufgrund ihrer bisher eher leichteren Beschwerden keinen Arzt aufsuchten und folglich auch nicht therapiert werden, ist der Unterschied zwischen berichteter und ärztlich bestätigter Krankheit (Differenz ca. 20\%) als problematisch zu bewerten, denn gerade beim Asthma lassen sich durch eine frühzeitige und ausreichende Therapie schwere Folgeschäden vermeiden oder reduzieren.

\section{"Die Ergebnisse des Bundes- Gesundheitssurveys 1998 sprechen im Einklang mit den Ergebnissen vieler anderer Untersuchungen der letzten Jahre für eine deuliche Zunahme allergischer Erkrankungen."}

Viele Ergebnisse wissenschaftlicher Untersuchungen aus den letzten 20 Jahren sprechen für eine Zunahme allergischer Erkrankungen $[16,17,18,19,20]$. Hinweise auf einen Anstieg der Allergiehäufigkeit lieferten für Westdeutschland bereits die Daten der drei Untersuchungswellen der Nationalen Gesundheitssurveys der DHP von 1984 bis 1991 [9]. Auf einen Prävalenzanstieg auch in Ostdeutschland deuteten erstmals Studienergebnisse bei Kindern hin. Zwischen 1991/92 und 1995/96 ist die Häufigkeit von Heuschnupfen bei neun- bis elfjährigen Kindern aus Leipzig von ca. $2 \%$ auf $5 \%$ gestiegen und die Sensibilisierungsrate (Prick-Test) von rund 19\% auf $27 \%$ [21]. Bei Erwachsenen stieg von 1990/92 bis 1998 nach den Daten des Bundes-Gesundheitssurveys die Häufigkeit von Heuschnupfen in Ostdeutschland von $6 \%$ auf $12 \%$ und in Westdeutschland von $11 \%$ auf $18 \%$. Obwohl in Ostdeutschland proportional weniger Menschen von Allergien betroffen sind, ist die relative Prävalenzzunahme hier höher als im Westen. Dies könnte ein Indiz für eine allmähliche Angleichung der Krankheitszahlen in Ost- und Westdeutschland sein.

\section{Literatur}

1. Hermann-Kunz E (1999) Heuschnupfenprävalenz in Deutschland - 0st-West-Vergleich und zeitlicher Trend. Gesundheitswesen 61 Sonderheft 2:S94-S99

2. Hermann-Kunz E (1999) Häufigkeit allergischer Krankheiten in Ost- und Westdeutschland. Gesundheitswesen 61 Sonderheft 2:S100-S105

3. Helbling A (1994) Nahrungsmittelallergie. Therapeutische Umschau 51:31-37

4. Wüthrich B (1995) Zur Nahrungsmittelallergie. Hautarzt 46:73-75
5. Bellach BM, Knopf H, Thefeld W (1998) Der Bundes-Gesundheitssurvey 1997/98. Gesundheitswesen 60 Sonderheft 2:S59-S68

6. Thefeld W, Stolzenberg H, Bellach BM (1999) Bundes-Gesundheitssurvey: Response, Zusammensetzung der Teilnehmer, NonResponder-Analyse. Gesundheitswesen 61 Sonderheft 2:S57-S61

7. Hoffmeister H, Bellach B-M (Hrsg) Die Gesundheit der Deutschen. RKI-Heft 7/1995

8. Nicolai T, von Mutius E (1996) Respiratory hypersensitivity and environmental factors: East and West Germany. Toxicology Letters 86: 105-113

9. Wiesner G, Todzy-Wolff I, Hoffmeister H (1994) Asthma bronchiale. In:Die Gesundheit der Deutschen:Ein Ost-West-Vergleich. SozEpHefte 4/1994:66-77

10. Wiesner G, Todzy-Wolff I, Hoffmeister H (1994) Heuschnupfen. In: Die Gesundheit der Deutschen: Ein Ost-West-Vergleich.SozEp-Hefte 4/1994:78-83

11. Nicolai T, Bellach B, von Mutius E, Thefeld W, Hoffmeister H (1997) Increased prevalence of sensitization against aeroallergens in adults in West compared with East Germany. Clinical and Experimental Allergy 27: 886-892

12. Wüthrich $B, S$ Shindler $C$, Leuenberger $P$ et al. (1995) Prevalence of Atopy and Pollinosis in the Adult Population of Switzerland (SAPALDIO Study). Int Arch Allergy Immunol 106:149-156

13. Neukirch F, Pin I, Knani J et al. (1995) Prevalence of asthma and asthma-like symptoms in three french cities. Respiratory Medicine 89:685-692

14. Jogi R, Janson C, Bjoernsson E et al. (1996) The prevalence of asthmatic respiratory symptoms among adults in Estonian and Swedish university cities. Allergy 51:331-336

15. Campello C, Ferrari M, Poli A et al. (1998) Prevalence of asthma and asthma-like symptoms in an adult population sample from Verona. ECRHS Verona. European Community Respiratory Health Survey. Monaldi Archives for Chest Disease 53:505-509

16. Burr ML (1993) The epidemiology of asthma. In: Burr ML (Hrsg) Epidemiology of clinical allergy. Karger AG, Basel 80-102

17. Schultz-Larsen F, Hanifin JM (1992) Secular change in the occurence of atopic dermatitis. Acta Derm Venereol 176:7-12

18. Sibbald B. (1993) The epidemiology of allergic rhinitis. In: Burr ML (Hrsg) Epidemiology of clinical allergy. Karger AG, Basel 61-79

19. Weiss KB, Gergen PJ, Wagener DK (1993) Breathing better or wheezing worse? The changing epidemiology of asthma morbidity and mortality. Ann Rev Pub Health 14: 491-513

20. Nowak D, Claussen M, Berger J, Magnussen $\mathrm{H}$ (1991) Weltweite Zunahme des Asthma bronchiale. Deutsches Ärzteblatt 88;36: B1951-B1955

21. von Mutius E, Weiland SK, Fritzsch C, Duhme H, Keil U (1998) Increasing prevalence of hay fever and atopy among children in Leipzig, East Germany. Lancet 351:862-866 